\title{
Suppression of murine melanoma growth by a vaccine of attenuated Salmonella carrying heat shock protein 70 and Herpes simplex virus-thymidine kinase genes
}

\author{
SHUGUANG ZENG ${ }^{1}$, JINCAI ZHANG ${ }^{1}$, JIREN ZHANG ${ }^{2}$, QICAI LIU $^{3}$, SUWEN WANG ${ }^{1}$, \\ SHIQING WU ${ }^{1}$, XIMAO PENG $^{1}$, JING SHAO $^{4}$ and WENGE HUANG ${ }^{5}$ \\ ${ }^{1}$ Oral and Maxillofacial Surgery, Guangdong Provincial Stomatological Hospital Affiliated to Southern Medical \\ University; ${ }^{2}$ Tumor Center, Pearl River Hospital Affiliated to Southern Medical University; ${ }^{3}$ Research Center \\ for Laboratory Medicine, Guangzhou Medical College; ${ }^{4}$ Lim Por Yen Center for Laboratory Medicine \\ and ${ }^{5}$ Research Center for Laboratory Animals, Sun Yat-Sen University, Guangzhou, P.R. China
}

Received September 12, 2011; Accepted October 17, 2011

DOI: $10.3892 / o r .2011 .1556$

\begin{abstract}
Attenuated Salmonella can invade tumor cells and acts as a eukaryotic expression vector for gene propagation. We constructed a bi-gene, eukaryotic co-expression DNA vaccine of Mycobacterium tuberculosis heat shock protein 70 (mtHSP70) and Herpes simplex virus-thymidine kinase (HSV-tk) and used attenuated Salmonella as a vector to treat murine melanoma. In vitro, recombinant Salmonella can carry plasmid stably and can invade into the cytoplasm of B16 tumor cells expressing the protein of the mtHSP70/HSV-tk gene by Western blot assay. In vivo, after the recombinant Salmonella was injected into tumors, the HSV-tk precursor drug ganciclovir (GCV) was administered to start the HSV-tk killing of tumor cells. We found that the mtHSP70/HSV-tk recombinant bacteria can raise $\mathrm{CD}^{+} \mathrm{T}$ lymphocytes in peripheral blood by flow cytometry and in tumor tissues by immunofluorescence detection, increase IFN- $\gamma$ contents in tumor tissue by ELISA and significantly suppress tumor growth.
\end{abstract}

\section{Introduction}

Gene vaccines are a new development in the field of cancer gene therapy (1). However, given the high polymorphism of major histocompatibility complex (MHC) antigens, the effectiveness of gene vaccines varies greatly among tumor patients.

Correspondence to: Dr Jincai Zhang, Guangdong Provincial Stomatological Hospital Affiliated to Southern Medical University, 366 Jiangnan Southern Road, Guangzhou 510280, P.R. China

E-mail: sunrisez@live.cn

Dr Jiren Zhang, Tumor Center, Pearl River Hospital Affiliated to Southern Medical University, Guangzhou 510282, P.R. China

E-mail: zhangdoctor2000@tom.com

Key words: recombinant DNA, cancer, vaccine, Salmonella, heat shock protein 70 , Herpes simplex virus-thymidine kinase
Weak antigenicity is also a drawback (2). Thermal effects, heat shock protein (HSP) promoter and HSP can all boost the efficacy of suicide genes (3-7). With strong antigenicity, Mycobacterium tuberculosis heat shock protein 70 (mtHSP70) has become an important immunological adjuvant. As one kind of antigenic vector molecule, it has so many epitopes of $\mathrm{B}$ cells and T cells as to elicit both cellular and humoral immunity (8-10). Attenuated Salmonella can invade tumor cells, has limited replication in tumor cells and has a high tropism for solid-organ tumors. It also acts as a eukaryotic expression vector for gene propagation and it is easy to be cleared by antibiotics compared to viral vectors (11-14). Malignant melanomas in the oral mucosa are the most malignant among all oral tumors. In this study, we constructed a bi-gene, eukaryotic co-expression DNA vaccine of mtHSP70 and Herpes simplex virus-thymidine kinase (HSV-tk) and employed the attenuated Salmonella as a vector to treat murine melanoma through an intratumoral injection.

\section{Materials and methods}

Animals, bacterial strains and cell line. All animal experiments were done in compliance with the NIH Guidelines for the Care and Use of Laboratory Animals, under license no. 0041126 issued by the Research Center for Laboratory Animals, Sun Yat-Sen University.

Sixty 6- to 8-week-old C57BL/6J mice with a mean body weight of $20 \mathrm{~g}$ were purchased from the Research Center for Laboratory Animals, Sun Yat-Sen University. Murine melanoma B16 cells were obtained from the Shanghai Cell Bank of the Chinese Academy of Science. The attenuated Salmonella typhimurium strain 7207 (courtesy of Dr Chen Hong, Shanghai Second Medical University) (15).

Construction of the mtHSP70 and HSV-tk gene eukaryotic expression plasmid. GenBank was accessed to retrieve the mRNA sequences of HSV-tk and mtHSP70 genes. The primer design software, DNA Club, was used to design and amplify 
the full-length cDNA-specific primer of genes HSV-tk and mtHSP70. The HSV-tk (GenBank no. J02224) primer sequences were forward 5'-GCGGCCGCGCGCCTTGTAGAAGCGC GTA-3', reverse 5'-GCGGCCGCCCTTCCGGTATTGTCTCC TTCC-3'. The upstream and downstream primers 5 ' were both introduced into the locus of the restriction endonuclease Not I (underlined sequence). The mtHSP70 (GenBank no. BX842573) primer sequences were forward 5'-CTCGAGCGCCTACATG GCTCGTGC-3', reverse 5'-GAATTCTTCACTTGGCCTC CCGG-3'. The upstream and downstream primers 5' were introduced into the loci of restrictive endonucleases $\mathrm{XhoI}$ and EcoRI (underlined sequence), respectively. The primer oligonucleotide chain was synthesized. Either the pcDNA3-tk plasmid (courtesy of Dr Qi-Cai Liu, Guangzhou Medical College) or the PVAX-mtHSP70-HSV2gD plasmid DNA (courtesy of Dr Jian-Yong Fan, Southern Medical University) was chosen as a template and the primer was made for each. The PrimeSTAR HS DNA polymerase (Takara Bio, Inc., Japan) was used for polymerase chain reaction (PCR). The HSV-tk and mtHSP70 gene cDNA fragments were harvested and recollected with a gel reagent kit (Qiagen, Inc., Germany). After adding an A-tailing at 3', the target gene fragments of $1192 \mathrm{bp}(\mathrm{tk})$ and $1898 \mathrm{bp}$ (mtHSP70) were linked to T vector pMD 18T (Takara Bio, Inc.). After transfection of E. coli TG1 (NEB, Inc., UK), the primers specific for genes HSV-tk and mtHSP70 gene were used for colony PCR. The positive recombinant plasmids pMD 18T-tk and pMD 18T-mtHSP70 were sequenced upstream and downstream and the results were compared with their homogenous gene sequences. The recombinant plasmids pMD 18T-tk and pMD 18T-mtHSP70, whose gene sequences were $100 \%$ homogenous and whose inserted restriction enzyme digestion loci were the same as the sequence recognized by a specific restriction enzyme, were employed to sub-clone the HSV-tk and mtHSP70 genes into PCMV-IRESEGFP (Invitrogen, Inc., USA). While constructing the plasmid PCMV-mtHSP70-IRES-TK, single NotI restriction enzyme digestion and double EcoRI and XhoI (NEB, Inc.) restriction enzyme digestion were conducted for restriction enzyme digestion analysis. In the meantime, the sub-cloning method was used to construct the control group plasmids pCMV-TKIRES-EGFP and pCMV-mtHSP70-IRES-EGFP. The corresponding restriction enzyme digestion system was constructed by double SalI and BamHI and EcoRI and XhoI (NEB, Inc.) for restriction enzyme digestion analysis.

$m R N A$ and protein expressions of recombinant plasmid $m t H S P 70 / H S V$-tk gene in B16 cells. B16 cells were cultured in 60-mm Petri dishes. Transfection was carried out according to the handling procedures specified in the Lipofectamine 2000 transfection reagent kit (Invitrogen, Inc.). Transfection efficiency was observed under fluorescence microscopy. The mRNA and protein expressions of the recombinant plasmid mtHSP70/HSV-tk gene in B16 cells were detected by RT-PCR and Western blot analysis. The mtHSP70 upstream specific primer was: 5'-CGTCTCGGTTCTGGAAGGTGGC-3'; the downstream specific primer was: 5'-ATTGAAGTAGGCGGG CGTCGTG-3'; the HSV-tk upstream specific primer was: 5'-ATATAGACGGTCCTCACGGG-3'; and the downstream specific primer was: 5'-TAATCCAGGATAAAGACGTGC-3'. The Western blot reagents of mtHSP70 were the mouse-derived
anti-mtHSP70 primary monoclonal $\mathrm{Ab}$ and the goat antimouse IgG secondary Ab (Santa Cruz Biotechnology, Inc., USA). The detection reagents of HSV-tk were the goat-derived anti-HSV-tk primary monoclonal $\mathrm{Ab}$ and rabbit anti-goat IgG secondary Ab (Santa Cruz Biotechnology, Inc.).

Recombinant Salmonella construct and its invasive effect on B16 cells. The competent Salmonella strain SL7207 was prepared (16). The plasmids of pCMV-mtHSP70-IRES-TK, pCMV-IRES-EGFP, pCMV-mtHSP70-IRES-EGFP and pCMV-TK-IRES-EGFP were transformed into Salmonella SL7207 (16) with an electroporator (Bio-Rad, USA). The corresponding recombinant Salmonella constructs were: SL7207/pCMV-mtHSP70-IRES-TK, SL7207/PCMVmtHSP70-IRES-EGFP, SL7207/PCMV-TK-IRES-EGFP and SL7207/PCMV-IRES-EGFP which were analyzed with restriction enzyme digestion. After restriction enzyme digestion analysis, 4 recombinant Salmonella constructs as above were inoculated onto the Luria-Bertani (LB) solid culture medium for 20 generations of repeated propagation and the plasmids were then extracted. The stability of the attenuated Salmonella SL7207 carrier plasmid was evaluated with restriction enzyme digestion analysis. In the meantime, B16 cells were mixed with the 4 Salmonella strains, respectively at a ratio of $1: 50$ and co-incubated at $37^{\circ} \mathrm{C}$ for $30 \mathrm{~min}$. The cells were washed twice with serum-free culture medium containing gentamicin $(50 \mathrm{mg} / \mathrm{l}$; Sigma, USA) to kill the extracellular bacteria. RPMI-1640 culture medium (Gibco, Inc., Australia) containing FBS $100 \mathrm{ml} / 1$ was incubated at $37^{\circ} \mathrm{C}$ for $4 \mathrm{~h}$. Tetracycline (Sigma) was added to reach a final mass concentration of $10 \mathrm{mg} / \mathrm{l}$ (17), which was further cultivated for $36 \mathrm{~h}$. The expression of EGFP was observed under a fluorescence microscope. Finally, the transfected B16 cells were observed under an electron microscopy for whether the recombinant Salmonella invading to B16 cells, and a Western blot assay was performed to detect the expression of the mtHSP70/HSV-tk protein.

Intratumoral injection of recombinant Salmonella against murine B16 tumors. The concentration of the murine melanoma strain of B16 cells was $10^{7} / \mathrm{ml}$. To establish the B16 tumor-bearing animal model, $100 \mu \mathrm{l}$ of B16 cell suspension fluid was injected subcutaneously on the right side of the backs of 60 pure-bred C57BL/6J mice. A vernier caliper was used to measure the tumor dimension once every 3 days. The tumor volume was calculated according to the formula (7): volume $=$ length $\mathrm{x}$ width ${ }^{2} \mathrm{x} 0.52$.

When the tumor volume reached $\sim 100 \mathrm{~mm}^{3}$, the animals were divided into 5 treatment groups of 12 tumorbearing mice each: i) an experiment group receiving intratumoral injections of recombinant Salmonella SL7207/ PCMV-mtHSP70-IRES-TK (Group HT); ii) control group 1 receiving intratumoral injections of recombinant Salmonella SL7207/PCMV-mtHSP70-IRES-EGFP (Group H); iii) control group 2 receiving intratumoral injections of recombinant Salmonella SL7207/PCMV-TK-IRES-EGFP (Group T); iv) control group 3 receiving intratumoral injection recombinant Salmonella SL7207/PCMV-IRES-EGFP (Group Sa); and v) control group 4 receiving intratumoral injections of PBS (Group PBS). In the experimental group and control groups H, 
A
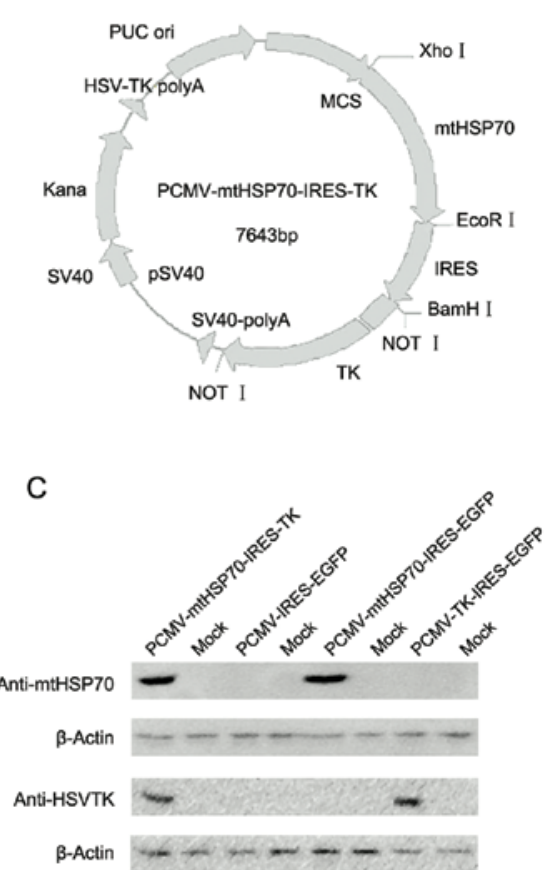

B

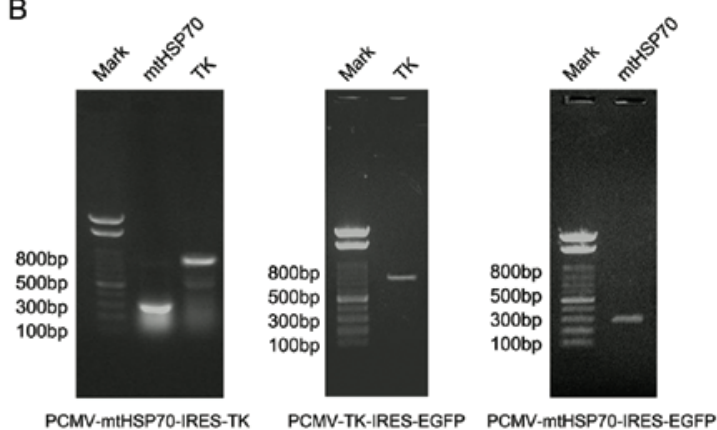

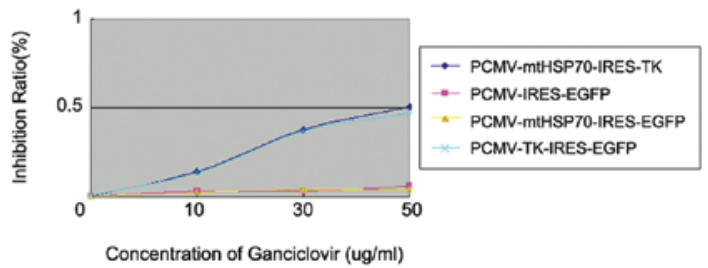

Figure 1. Construction of the mtHSP70 and HSV-tk gene eukaryotic expression plasmid and MTT assays. (A) Structure of the pCMV-mtHSP70-IRES-TK plasmid containing the sequence of mtHSP70 and HSV-tk. (B) mRNA and protein expressions of the recombinant plasmid mtHSP70/HSV-tk gene in B16 cells as detected by RT-PCR and (C) Western blot analysis. (D) Results of 6 separate MTT assays showing that the in vitro tumor-killing effect of plasmids containing the tk gene over B16 cells increased proportionally with the concentration of ganciclovir. The inhibition ratio was calculated. At a concentration of $50 \mu \mathrm{g} / \mathrm{ml}$, the in vitro tumor-killing ratios of recombinant plasmids pCMV-mtHSP70-IRES-TK (blue curve) and pCMV-TK-IRES-EGFP (green curve) were 50.45 and $46.96 \%$, respectively. These values differed significantly from those for pCMV-IRES-EGFP (red curve) and pCMV-mtHSP70-IRES-EGFP (yellow curve), $\mathrm{P}=0.000$ vs. $\mathrm{P}<0.05$.

$\mathrm{T}$ and $\mathrm{Sa}, 100 \mu \mathrm{l}$ of $10^{9} \mathrm{CFU} / \mathrm{ml}$ recombinant Salmonella was injected into the tumor at two sites; in control group PBS, $100 \mu 1$ PBS was injected into the tumor at two sites. Recombinant bacterium or PBS was injected once. GCV $50 \mathrm{mg} / \mathrm{kg}$ dissolved in $0.5 \mathrm{ml}$ PBS was injected intraperitoneally twice daily for 5 consecutive days, beginning at $24 \mathrm{~h}$ after injection of recombinant Salmonella. This schedule was counted as one course, and three consecutive courses were administered. A vernier caliper was used to measure the long and short diameters of the tumors once in each group every 3 days. At the end of the third course, the tumor-inhibiting rate of each group was calculated according to the measured tumor volume.

Flow cytometry profiles of $\mathrm{CD}^{+} / \mathrm{CD}^{+}$and $\mathrm{CD}^{+} / C D 8^{+}$in $T$ lymphocytes and NK cells. At the end of the third course, $0.6 \mathrm{ml}$ of peripheral blood was drawn from the orbital region of 5 tumor-bearing mice from each group and put into an anticoagulant tube. The profiles of $\mathrm{CD}^{+} / \mathrm{CD}^{+}, \mathrm{CD}^{+} / \mathrm{CD}^{+}$and $\mathrm{CD}^{+} / \mathrm{CD}^{2} 6^{+}$in $\mathrm{T}$ lymphocytes and NK cells were analyzed with flow cytometry using fluorescently tagged rat anti-mouse antibodies of CD8PE, CD4PE, CD3FITC and CD56PE (Becton-Dickinson, Inc., USA).

ELISA assay of IFN- $\gamma$ levels in tumor tissues. Five tumorbearing mice were sacrificed by decapitation after peripheral blood was drawn. The tumor was extracted and weighed and the mean tumor-inhibition rate was calculated for each group. Each tumor sample was divided into three parts and prepared
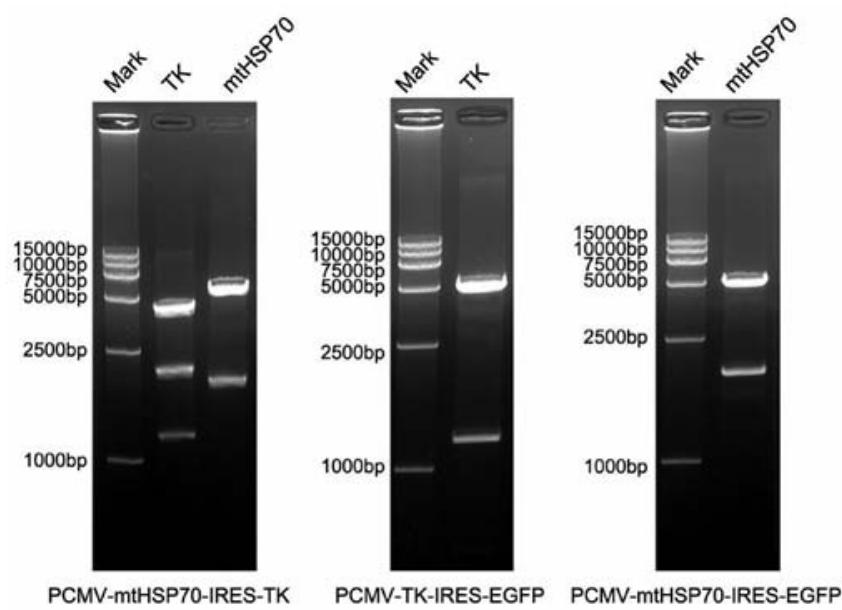

Figure 2. Restriction enzyme verification on recombinant plasmid pCMVmtHSP70-IRES-TK by NotI and EcoRI+XhoI (left); restriction enzyme verification on recombinant plasmid pCMV-TK-IRES-EGFP by SalI and BamHI (middle); restriction enzyme verification on recombinant plasmid pCMV-mtHSP70-IRES-EGFP by XhoI and EcoRI (right).

into a homogenate of $50 \mathrm{mg}$ tissue $/ \mathrm{ml}$. The homogenate was centrifuged at $12000 \mathrm{x} \mathrm{g}$ at $4^{\circ} \mathrm{C}$ for $15 \mathrm{~min}$. After centrifugation, the supernatant was collected for ELISA. The level of IFN- $\gamma$ in tumor tissue was assayed according to the instructions of the murine IFN- $\gamma$ ELISA reagent kit (Sigma). 

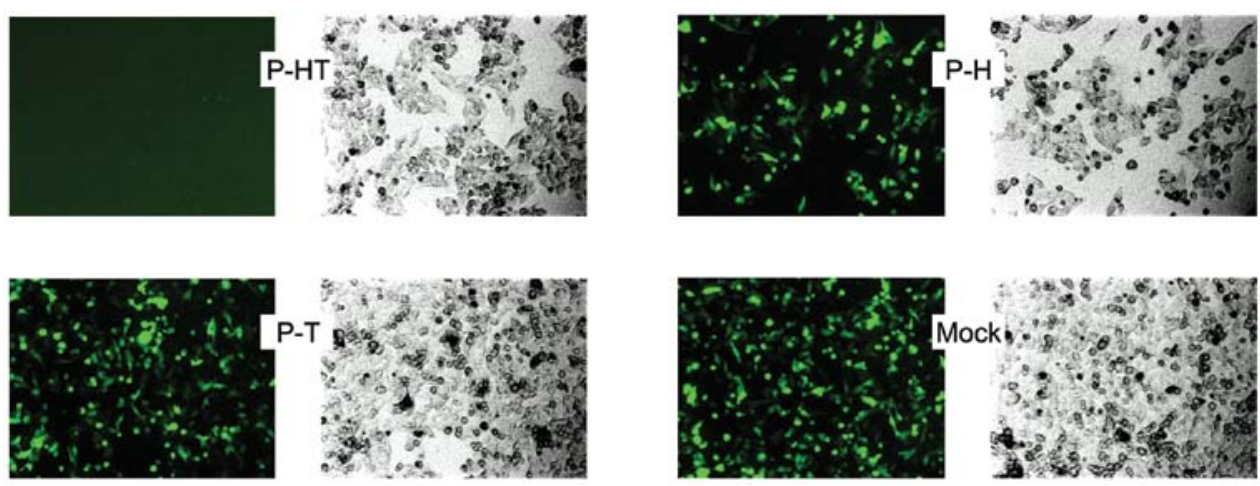

Figure 3. Fluorescent image of B16 cells transfected by PCMV-mtHSP70-IRES-TK(P-HT), PCMV-mtHSP70-IRES-EGFP(P-H), PCMV-TK-IRESEGFP(P-T), PCMV-IRES-EGFP (mock); magnification x200.

A
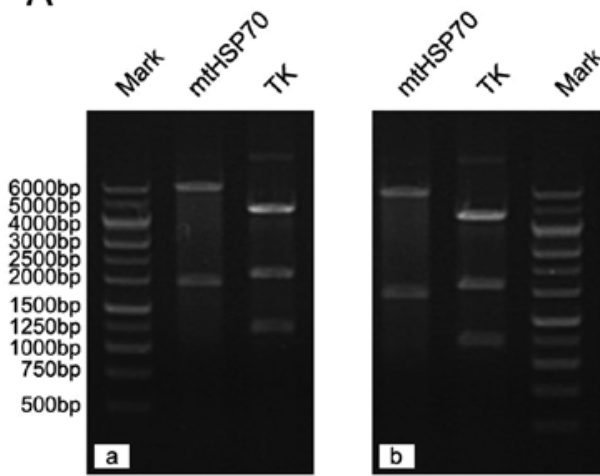

SL7207 / PCMV-mtHSP70-IRES-TK
B

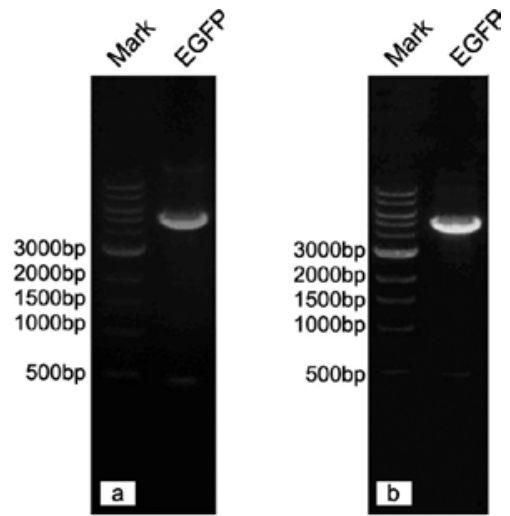

SL7207 / PCMV-IRES-EGFP
C

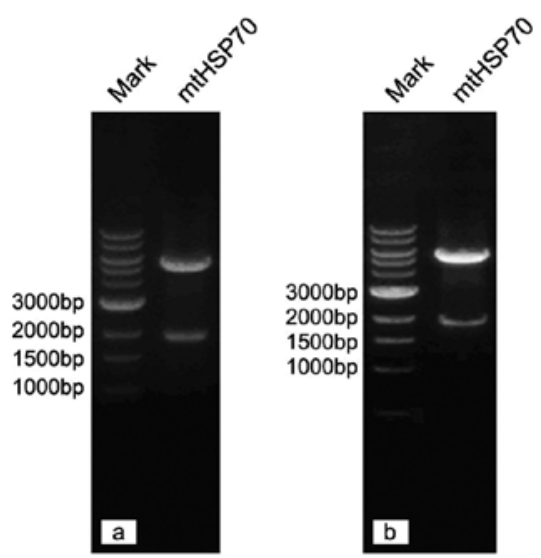

SL7207 / PCMV-mtHSP70-IRES-EGFP
D

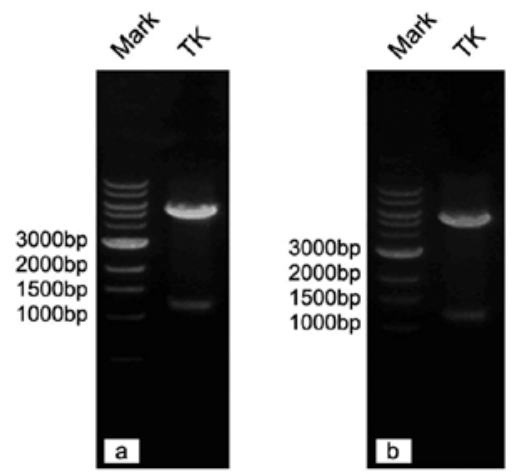

SL7207 / PCMV-TK-IRES-EGFP

Figure 4. (A) Plasmids extracted from recombinant Salmonella typhimurium SL7207/PCMV-mtHSP70-IRES-TK underwent restriction enzyme verification by single restriction enzyme of NotI (tk) and double restriction enzymes of EcoRI and XhoI (mtHSP70), respectively, (A-a) first passage; (A-b) after 20 passages. (B) Plasmids extracted from recombinant Salmonella typhimurium SL7207/PCMV-IRES-EGFP underwent restriction enzyme verification by single restriction enzyme of KpnI, (B-a) first passage; (B-b) after 20 passages. (C) Plasmids extracted from recombinant Salmonella typhimurium SL7207/ PCMV-mtHSP70-IRES-EGFP underwent restriction enzyme verification by double restriction enzymes of EcoRI and XhoI, (C-a) first passage; (C-b) after 20 passages. (D) Plasmids extracted from recombinant Salmonella typhimurium SL7207/PCMV-TK-IRES-EGFP underwent restriction enzyme verification by double restriction enzymes of SalI and BamHI, (D-a) first passage; (D-b) after 20 passages.

Immunofluorescence distribution of $C D 8^{+}$lymphocytes in tumors. Tumor tissue was fixed with $10 \%$ formalin, embedded in paraffin, stained with $\mathrm{H} \& \mathrm{E}$ and mounted on slides. According to the instructions of the immunofluorescence detection reagent kit, rat anti-mouse CD8PE antibody with a red fluorescent tag was used to analyze the distribution of $\mathrm{CD}^{+}$lymphocytes in the tumor (Becton-Dickinson, Inc.).

Electron microscopic evaluation of morphological changes in tumor cells. The prefixation fluid was used to fix tumor tissue 

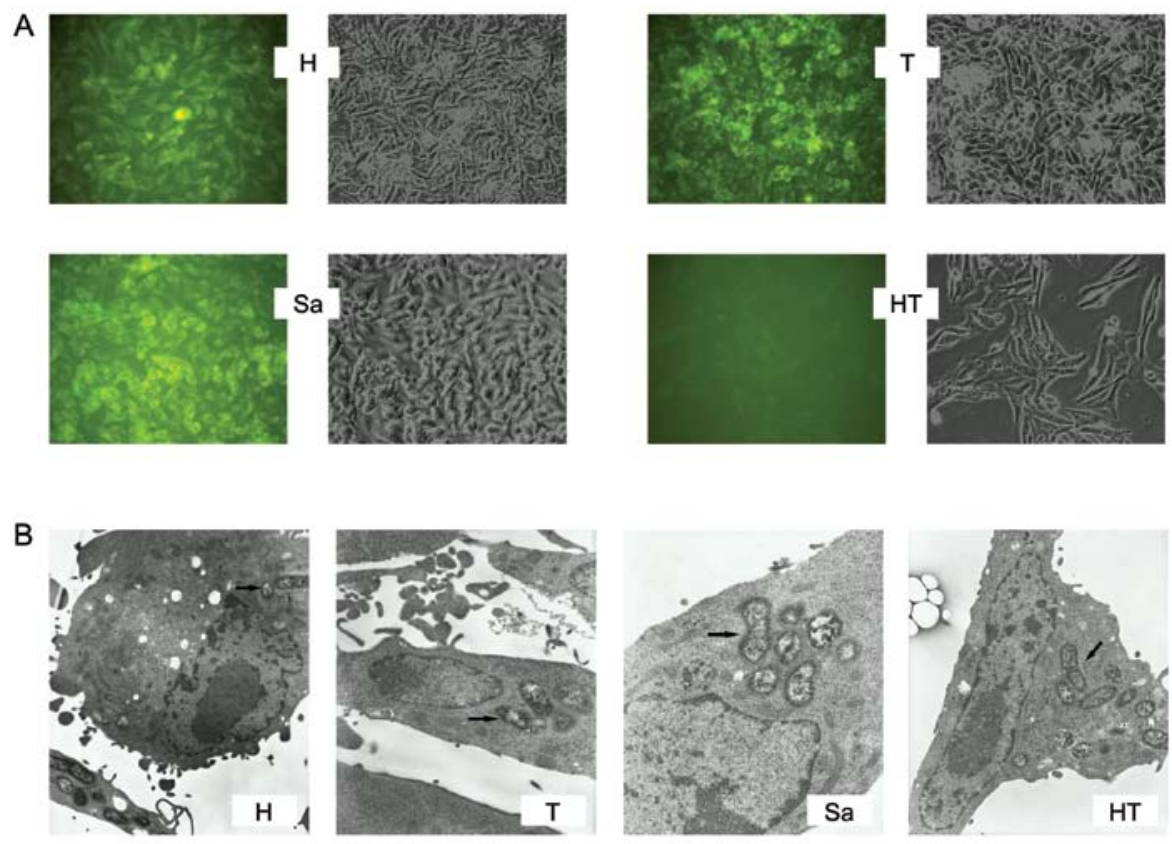

C
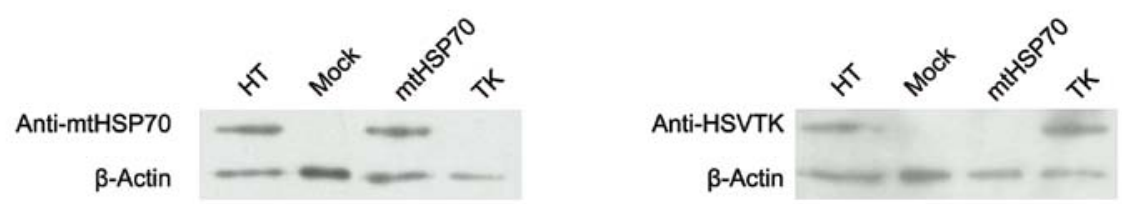

Figure 5. Recombinant Salmonella construct and its invasive effect on B16 cells. (A) Under fluorescence microscopy $36 \mathrm{~h}$ after transfection. The recombinant bacteria containing EGFP expressed a strong green fluorescent protein (photos on the left; photos on the right were taken under visible light; magnification x200). The expression rate was around 95\%. (HT, SL7207/PCMV-mtHSP70-IRES-TK; H, SL7207/PCMV-mtHSP70-IRES-EGFP; T, SL7207/PCMV-TKIRES-EGFP; Sa, SL7207/PCMV-IRES-EGFP). (B) Electron micrographs of recombinant Salmonella typhimurium infecting murine melanoma B16 cells. Multiple bacteria (arrows) are present in the cytoplasm of the cells. (C) Western blot analysis revealed that recombinant bacterial transfected B16 cells expressed the relevant proteins of the target genes. HT, SL7207/PCMV-mtHSP70-IRES-TK; mtHSP70, SL7207/PCMV-mtHSP70-IRES-EGFP; TK, SL7207/ PCMV-TK-IRES-EGFP; Mock, SL7207/PCMV-IRES-EGFP.

for morphological observation under electron microscopy to show the influence of recombinant vaccine on tumor cells and the tumor microenvironment.

\section{Results and Discussion}

Vector construction and cell transfection expression. Restriction enzyme digestion analysis confirmed the successful construction of the recombinant plasmids (Fig. 1A): pCMV-mtHSP70-IRES-TK, pCMV-TK-IRES-EGFP and pCMV-mtHSP70-IRES-EGFP (Fig. 2); pCMV-IRES-EGFP was taken as blank control. The above plasmids and the recombinant plasmid containing EGFP were transfected into B16 cells. After $48 \mathrm{~h}$, the cell expressed a green fluorescence of around $70 \%$ (Fig. 3). Within the RT-PCR reaction product of the recombinant plasmid transfected B16 cells, a product identical in size to the target gene primer was found (Fig. 1B). The Western blot analysis showed that the pCMV-mtHSP70IRES-TK plasmid transfected B16 cells expressed the mtHSP70 and HSV-tk proteins. The mtHSP70/HSV-tk plasmid also expressed the mtHSP70 and HSV-tk proteins (Fig. 1C).

Construction of the recombinant Salmonella strains and electron microscopic observations of its invasiveness in B16 cells.
The plasmid was extracted from the recombinant Salmonella and restriction enzyme digestion analysis was conducted for the corresponding restriction enzyme digestion system. Electrophoresis of all restriction enzyme digestion products revealed a specific map of restriction enzyme digestion, which indicated that the recombinant Salmonella was successfully constructed. After restriction enzyme digestion analysis, four recombinant Salmonella: SL7207/pCMV-mtHSP70IRES-TK, SL7207/PCMV-mtHSP70-IRES-EGFP, SL7207/ PCMV-TK-IRES-EGFP and SL7207/PCMV-IRES-EGFP were inoculated respectively onto the Luria-Bertani (LB) solidculture medium for 20 generations of repeated propagation, after which the plasmids were extracted. The corresponding restriction enzyme digestion system was used for analysis. As expected, the results indicated that the carrier plasmid of the recombinant Salmonella as above was stable (Fig. 4). After transfection with the recombinant bacteria, the B16 cells appeared rounded under light microscopy. At $36 \mathrm{~h}$ after transfection, the recombinant bacterium containing EGFP expressed a strong green fluorescent signal under fluorescence microscopy and the expression rate was around 95\% (Fig. 5A). Under electron microscopy, the cytoplasm of the B16 tumor cells was observed to have been invaded by multiple recombinant bacteria. The cells were also swollen (Fig. 5B). Western 
Table I. Tumor growth characteristics in C57BL/6J mice transfected with murine melanoma B16 cells and treated with a bi-gene, eukaryotic co-expression DNA vaccine of Mycobacterium tuberculosis heat shock protein 70 and Herpes simplex virus-thymidine kinase administered with attenuated Salmonella as a vector.

\begin{tabular}{|c|c|c|c|c|c|c|}
\hline & \multicolumn{5}{|c|}{ Treatment group } & \multirow[b]{2}{*}{ P-value } \\
\hline & HT & $\mathrm{H}$ & $\mathrm{T}$ & $\mathrm{Sa}$ & PBS & \\
\hline \multicolumn{7}{|c|}{ Tumor volume (mean $\pm \mathrm{SD}), \mathrm{mm}^{3}$} \\
\hline Before treatment & $101.54 \pm 4.52$ & $101.54 \pm 4.36$ & $100.37 \pm 3.74$ & $100.18 \pm 6.41$ & $97.27 \pm 10.95$ & $0.528>0.05$ \\
\hline After treatment & $1366.77 \pm 401.15^{\mathrm{a}}$ & $2351.34 \pm 819.08^{\mathrm{b}}$ & $2511.09 \pm 809.11^{\mathrm{c}}$ & $2902.58 \pm 621.52^{\mathrm{d}}$ & $6958.62 \pm 1200.40^{\mathrm{e}}$ & $0.000<0.05$ \\
\hline \multicolumn{7}{|c|}{ Tumor-inhibition rate by volume, $\%^{\mathrm{e}}$} \\
\hline After treatment & 80.3 & 66.2 & 63.9 & 58.3 & - & \\
\hline \multicolumn{7}{|c|}{ Tumor weight (mean $\pm \mathrm{SD}$ ), g } \\
\hline Post-mortem & $1.9586 \pm 1.1095$ & $3.0433 \pm 1.6854$ & $3.3943 \pm 1.5035$ & $3.9327 \pm 0.6607$ & $8.9173 \pm 1.1803$ & $0.000<0.05$ \\
\hline \multicolumn{7}{|c|}{ Tumor-inhibition rate by weight, $\%$} \\
\hline After treatment & 78.0 & 65.9 & 61.9 & 55.9 & - & \\
\hline
\end{tabular}
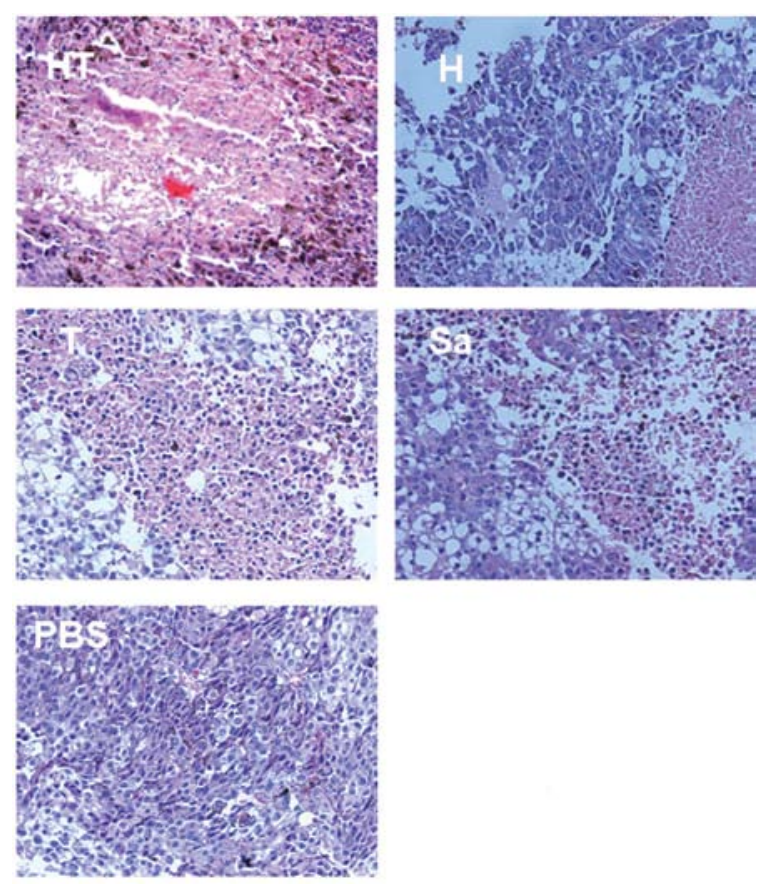

Figure 6. Pathological analysis. The H\&E-stained tumor slides show large patches of necrosis in Group HT. In Groups H, T and Sa, pathologica analysis showed local necrosis lesions. In Group PBS, the tumor tissue shows no marked cellular changes (H\&E; magnification x200).

blot analysis revealed that all recombinant bacterial transfected B16 cells expressed the relevant proteins of the target genes (Fig. 5C). Sizemore and colleagues (18) proposed that intracellular bacterium should be the third inoculation method of nucleic acid vaccine. As one kind of invasive intracellular bacteria, Salmonella is a gram-negative intestinal pathogen
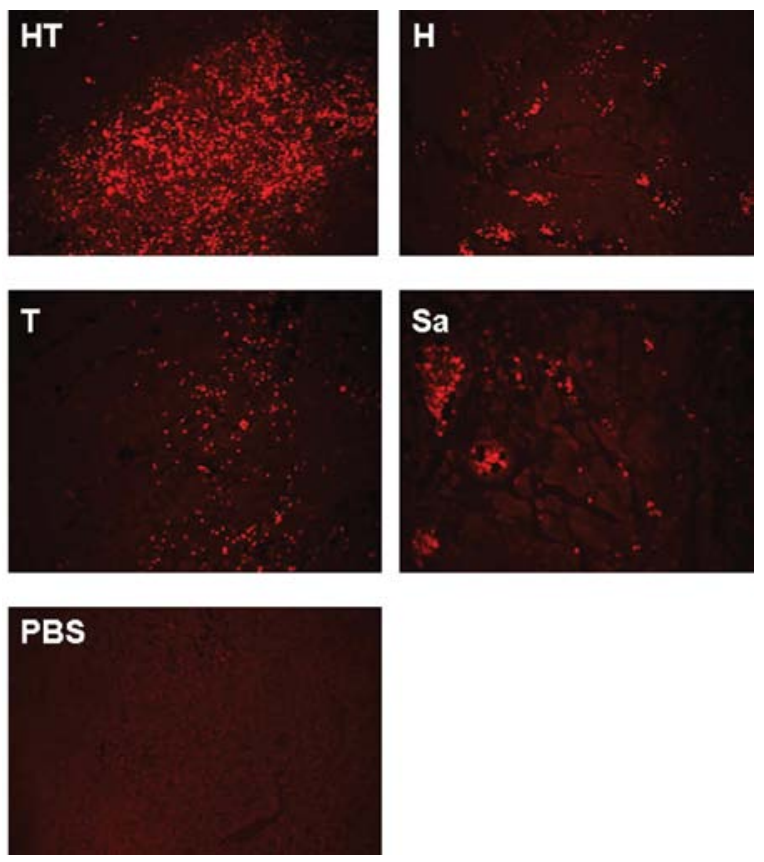

Figure 7. $\mathrm{CD} 8^{+} \mathrm{T}$ lymphocytes detected by immunofluorescence in tumor tissue. Large quantities of $\mathrm{CD}^{+} \mathrm{T}$ lymphocytes (red) were detected by immunofluorescence in tumors after treatment with the recombinant vaccine in Group HT, and some appear in Group H, T and Sa, but no $\mathrm{CD}^{+} \mathrm{T}$ lymphocytes were detected in Group PBS (magnification x200).

(19) that was the first recombinant bacterial vector for antigenic presentation (20,21). Attenuated Salmonella can invade tumor cells, although its replication in tumor cells is limited and it has a high level of tropism for solid-organ tumors $(11,12)$. It has also been employed as a eukaryotic expression vector for gene delivery (13). Its interaction with the host is mediated 
Table II. Antitumor mechanisms of the recombinant Salmonella vaccine.

\begin{tabular}{|c|c|c|c|c|c|c|}
\hline & \multicolumn{5}{|c|}{ Treatment group } & \multirow[b]{2}{*}{ P-value } \\
\hline & HT & $\mathrm{H}$ & $\mathrm{T}$ & $\mathrm{Sa}$ & PBS & \\
\hline \multicolumn{7}{|c|}{ Cells in peripheral blood samples, $\mathrm{n}^{\mathrm{a}}$} \\
\hline $\mathrm{CD}^{+} / \mathrm{CD}^{+}$ & $31.8 \pm 8.0$ & $34.2 \pm 4.2$ & $29.9 \pm 6.3$ & $27.1 \pm 5.3$ & $30.4 \pm 1.9$ & 0.384 \\
\hline $\mathrm{CD}^{+} / \mathrm{CD}^{+}{ }^{+}$ & $41.7 \pm 12.1^{\mathrm{b}}$ & $26.2 \pm 2.3$ & $23.5 \pm 1.4$ & $23.8 \pm 1.3$ & $20.4 \pm 1.7$ & $0.005<0.05$ \\
\hline NK & $20.4 \pm 15.6$ & $19.0 \pm 11.0$ & $15.7 \pm 9.2$ & $14.1 \pm 5.3$ & $16.2 \pm 5.4$ & 0.857 \\
\hline IFN- $\gamma, \mathrm{pg} / \mathrm{ml}^{\mathrm{a}}$ & $150.4098 \pm 58.2892^{\mathrm{b}}$ & $67.6573 \pm 24.3157$ & $57.7418 \pm 36.9552$ & $42.5049 \pm 29.2696$ & $25.1185 \pm 11.6308$ & $0.000<0.05$ \\
\hline
\end{tabular}

${ }^{a}$ Values are expressed as means \pm SD. ${ }^{b}$ Group HT differed significantly from all other groups at the 0.05 level. HT, SL7207/PCMV-mtHSP70-IRES-TK; H, SL7207/PCMV-mtHSP70-IRES-EGFP; T, SL7207/PCMV-TK-IRES-EGFP; Sa, SL7207/PCMV-IRES-EGFP.
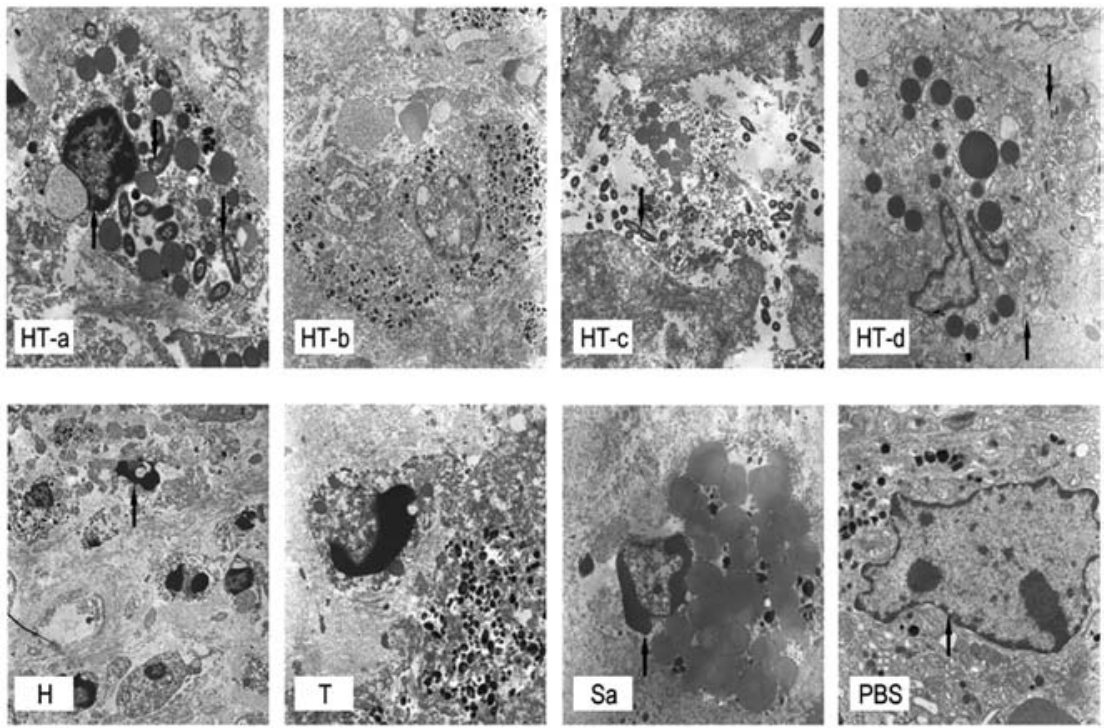

Figure 8. Electron microscopic evaluation. Electron micrographs showing that a large number of recombinant bacteria (HT-a, down arrow) has invaded the tumor cells. These cells show pyknosis, perinuclear cellular apoptosis (HT-a, up arrow), endoplasmic reticulum swelling, flocculent changes in the mitochondrial cristae and cellular lysis and necrosis (HT-b). Bacteria in the cytoplasm of tumor cells undergoing mitotic replication (HT-c, down arrow), which lyses the tumor cells. Group HT has specialized macrophages, which have a rich cytoplasmic lysosome pseudopodia (HT-d, up arrow) to capture tumor cells with melanin particles (HT-d, down arrow). Tumor cellular apoptosis observed in Groups T, H and Sa (up arrows); Group PBS shows no obvious changes in tumor cells.

by a Type III secreting mechanism, through which the bacterial protein is transferred into eukaryotic cells $(22,23)$. Some researchers believe that the release of DNA vaccine generally occurs after the natural autolysis of the recombinant bacterium carrying the DNA vaccine (24).

Intratumoral injection of recombinant Salmonella against murine melanoma. Tumor volume was not significantly different among groups at the start of treatment but was different after treatment (Table I). Pairwise comparisons showed that the mean tumor volume in Group HT was significantly lower than those of all other groups (Table I). Mean tumor volumes in Groups H, T and Sa did not differ from each other but were significantly different from the HT and PBS groups. Tumor growth was slow in Group HT, somewhat suppressed in Groups $\mathrm{H}, \mathrm{T}$ and $\mathrm{Sa}$, and fast in Group PBS (Table I). Group HT had the highest mean rate of tumor inhibition by volume, at $80.3 \%$. Group HT also had the lowest mean tumor weight, and the highest mean rate of tumor inhibition by weight, at $78 \%$ (Table I). The results of tumorinhibition calculated by weight were essentially the same as those when calculated by volume. Our results indicate that the tumor-inhibiting effects of HT recombinant bacteria were superior to those of all control groups. Among control groups, the recombinant bacteria containing mtHSP70 or the tk gene, and attenuated Salmonella, all had some antitumor effect, as reported in similar studies $(14,17,25)$. The hypoxic environment of solid-organ tumors facilitates the accumulation and multiplication of Salmonella. When employed as the inducing vector of a gene vaccine, the attenuated Salmonella cannot only replicate in the target tumor, it also expresses a specific in situ antitumor protein. Because it competes with the tumor for nutrients, it has direct inhibiting effects of tumor growth. Salmonella enters into tumor cells and causes their death. The self-generated immunological response of Salmonella did not inhibit that of the vaccine $(26,27)$. Under the effect 
of $\mathrm{GCV}$, the tk gene in the recombinant bacteria provides a certain killing effect. Therefore, in the control groups, the recombinant bacteria containing mtHSP70 or the tk gene and attenuated Salmonella all had some antitumor effect.

We detected the distribution of $\mathrm{CD}^{+} / \mathrm{CD}^{+}$; $\mathrm{CD}^{+} / \mathrm{CD}^{+} \mathrm{T}$ lymphocytes and NK cells in peripheral blood samples with flow cytometry (Table II). Between-group differences for the $\mathrm{CD}^{+} / \mathrm{CD}^{+} \mathrm{T}$ lymphocytes were significant $(\mathrm{P}<0.05)$. The number of $\mathrm{CD}^{+} / \mathrm{CD}^{+} \mathrm{T}$ lymphocytes in Group HT was markedly higher than that in all control groups $(\mathrm{P}<0.05)$. But between-group differences for $\mathrm{CD}^{+} / \mathrm{CD}^{+}$and $\mathrm{NK}$ cells, were not significant $(\mathrm{P}>0.05)$.

The IFN- $\gamma$ contents of the tumor tissue were detected by ELISA. The variances in the levels of IFN- $\gamma$ differed greatly and so were analyzed with the Welch test, which found that between-group differences were significant (H statistic, 25.639, $\mathrm{P}<0.001)$. The mean level of the IFN- $\gamma$ in Group HT was markedly higher than those of all control groups (Table II). IFN- $\gamma$ is the most characteristic cytokine secreted by Th1 cells. It is mainly produced by activated $\mathrm{T}$ cells (including Th0, Th1 and all $\mathrm{CD}^{+}$) and NK cells. The functions of IFN- $\gamma$ are activating macrophages and enhancing their functions, promoting multiple cells to express MHC I and II molecules, prompting the cellular differentiation of Th0 into Th1, suppressing the cellular proliferation of $\mathrm{Th} 2$, stimulating the maturity and activity of cytotoxic T lymphocytes and promoting the differentiation of B cells (28).

Pathological analysis of the H\&E-stained tumor slides revealed large patches of necrosis in Group HT. In Groups H, $\mathrm{T}$ and Sa, pathological analysis showed local necrosis lesion. In Group PBS, the tumor tissue showed no marked cellular changes (Fig. 6). Direct immunofluorescence with the CD8 antibody tagged with red fluorescent phycoerythrin showed a large number of $\mathrm{CD}^{+} \mathrm{T}$ lymphocytes within tumor tissue in Group HT. Only a few of $\mathrm{CD}^{+} \mathrm{T}$ lymphocytes in tumor tissues or within vascular in Groups $\mathrm{H}, \mathrm{T}$ and $\mathrm{Sa}$ but not in Group PBS (Fig. 7). Electron microscopic evaluation showed that large numbers of recombinant bacteria had invaded the tumor cells. Within the cytoplasm, the bacteria underwent mitotic replication and lysed the tumor cells. In Group HT, the tumor cells showed pyknosis, perinuclear cellular apoptosis, endoplasmic reticulum swelling, flocculent changes of mitochondrial cristae and cellular lysis and necrosis. Pyknosis and perinuclear cellular apoptosis might were sometimes observed in Groups T, H and Sa; tumor cells in Group PBS had no obvious changes when observed under electron microscopy. In Group HT, specialized macrophages, which had a rich supply of cytoplasmic lysosomes, were extending pseudopodia for capturing tumor cells with melanin particles (Fig. 8). This observation indicates that the HT vaccine maybe activates antigen-presenting cells. Macrophages express a large variety of surface markers, such as MHC (types I and II) antigen, some adhesion and co-stimulatory molecules, complement receptors and Toll-like receptors (29).

In summary, the results of this research show that attenuated Salmonella not only acts as a eukaryotic expression vector for gene propagation but also has a synergistic antitumor effect with mtHSP70/HSV-tk genes. The recombinant DNA vaccine of attenuated Salmonella carrying eukaryotic mtHSP70/ HSV-tk co-expression genes can markedly raise $\mathrm{CD} 8^{+} \mathrm{T}$ lymphocytes in peripheral blood and in tumor tissues, increase IFN- $\gamma$ contents in tumor tissue and significantly suppress tumor growth.

\section{Acknowledgements}

This study was supported by a grant from Guangdong Provincial Science and Technology Plan Project (2009B030801264).

\section{References}

1. Srivastava IK and Liu MA: Gene vaccines. Ann Intern Med 138: 550-559, 2003.

2. Triozzi PL, Aldrich W, Allen KO, Carlisle RR, LoBuglio AF and Conry RM: Phase I study of a plasmid DNA vaccine encoding MART-1 in patients with resected melanoma at risk for relapse. J Immunother 28: 382-388, 2005.

3. Young JG, Green NK, Mautner V, Searle PF, Young LS and James ND: Combining gene and immunotherapy for prostate cancer. Prostate Cancer Prostatic Dis 11: 187-193, 2008.

4. Somoto H, Ohtsuru A, Braiden V, et al: Heat-directed suicide gene therapy mediated by heat shock protein promoter for gastric cancer. Oncol Rep 15: 629-635, 2006.

5. Lee YJ, Lee H and Borrelli MJ: Gene transfer into human prostate adenocarcinoma cells with an adenoviral vector: hyperthermia enhances a double suicide gene expression, cytotoxicity and radiotoxicity. Cancer Gene Ther 9: 267-274, 2002.

6. Parry JJ, Sharma V, Andrews R, Moros EG, Piwnica-Worms D and Rogers BE: PET imaging of heat-inducible suicide gene expression in mice bearing head and neck squamous cell carcinoma xenografts. Cancer Gene Ther 16: 161-170, 2009.

7. Ren W, Strube R, Zhang X, Chen SY and Huang XF: Potent tumor-specific immunity induced by an in vivo heat shock protein-suicide gene-based tumor vaccine. Cancer Res 64: 6645-6651, 2004.

8. Wendling U, Paul L, van der Zee R, Prakken B, Singh M and van Eden W: A conserved mycobacterial heat shock protein (hsp) 70 sequence prevents adjuvant arthritis upon nasal administration and induces IL-10-producing T cells that cross-react with the mammalian self-hsp70 homologue. J Immunol 164: 2711-2717, 2000.

9. Wells $\mathrm{AD}$ and Malkovsky M: Heat shock proteins, tumor immunogenicity and antigen presentation: an integrated view. Immunol Today 21: 129-132, 2000.

10. Gaston JS: Heat shock proteins as potential targets in the therapy of inflammatory arthritis. Biotherapy 10: 197-203, 1998.

11. Pawelek JM, Low KB and Bermudes D: Bacteria as tumourtargeting vectors. Lancet Oncol 4: 548-556, 2003.

12. Cunningham $C$ and Nemunaitis J: A phase I trial of genetically modified Salmonella typhimurium expressing cytosine deaminase (TAPET-CD, VNP20029) administered by intratumoral injection in combination with 5-fluorocytosine for patients with advanced or metastatic cancer. Hum Gene Ther 12: 1594-1596, 2001.

13. Agorio C, Schreiber F, Sheppard M, et al: Live attenuated Salmonella as a vector for oral cytokine gene therapy in melanoma. J Gene Med 9: 416-423, 2007.

14. Pawelek JM,Low KB and Bermudes D: Tumor-targeted Salmonella as a novel anticancer vector. Cancer Res 57: 4537-4544, 1997.

15. Hoiseth SK and Stocker BA: Aromatic-dependent Salmonella typhimurium are non-virulent and effective as live vaccines. Nature 291: 238-239, 1981.

16. Zhao M, Yang M, Ma H, et al: Targeted therapy with a Salmonella typhimurium leucine-arginine auxotroph cures orthotopic human breast tumors in nude mice. Cancer Res 66: 7647-7652, 2006.

17. Avogadri F, Martinoli C, Petrovska L, et al: Cancer immunotherapy based on killing of Salmonella-infected tumor cells. Cancer Res 65: 3920-3927, 2005.

18. Sizemore DR, Branstrom AA and Sadoff JC: Attenuated bacteria as a DNA delivery vehicle for DNA-mediated immunization. Vaccine 15: 804-807, 1997.

19. Figueroa-Bossi N, Uzzau S, Maloriol D and Bossi L: Variable assortment of prophages provides a transferable repertoire of pathogenic determinants in Salmonella. Mol Microbiol 39: 260-271, 2001 
20. Curtiss R III: Bacterial infectious disease control by vaccine development. J Clin Invest 110: 1061-1066, 2002.

21. Theys J, Barbé S, Landuyt W, et al: Tumor-specific gene delivery using genetically engineered bacteria. Curr Gene Ther 3: 207-221, 2003.

22. Kitamura S, Kondo S, Shinomura Y, et al: Expression of hepatocyte growth factor and c-met in ulcerative colitis. Inflamm Res 49: 320-324, 2000

23. Platt J, Sodi S, Kelley M, et al: Antitumour effects of genetically engineered Salmonella in combination with radiation. Eur J Cancer 36: 2397-2402, 2000.

24. Darji A, Guzmán CA, Gerstel B, et al: Oral somatic transgene vaccination using attenuated S. typhimurium. Cell 91: 765-775, 1997.

25. Shilling DA, Smith MJ, Tyther R, et al: Salmonella typhimurium stimulation combined with tumour-derived heat shock proteins induces potent dendritic cell anti-tumour responses in a murine model. Clin Exp Immunol 149: 109-116, 2007.
26. Vassaux G, Nitcheu J, Jezzard S and Lemoine NR: Bacterial gene therapy strategies. J Pathol 208: 290-298, 2006.

27. Hummel S, Apte RN, Qimron U, Vitacolonna M, Porgador A and Zöller M: Tumor vaccination by Salmonella typhimurium after transformation with a eukaryotic expression vector in mice: impact of a Salmonella typhimurium gene interfering with MHC class I presentation. J Immunother 28: 467-479, 2005.

28. Smyth MJ, Hayakawa Y, Takeda K and Yagita H: New aspects of natural-killer-cell surveillance and therapy of cancer. Nat Rev Cancer 2: 850-861, 2002.

29. Roger T, David J, Glauser MP and Calandra T: MIF regulates innate immune responses through modulation of Toll-like receptor 4. Nature 414: 920-924, 2001. 